\title{
Every 3-equivalenced association scheme is Frobenius
}

\author{
Mitsugu Hirasaka • Kyoung-Tark Kim • \\ Jeong Rye Park
}

Received: 3 October 2013 / Accepted: 12 May 2014 / Published online: 28 May 2014

(C) Springer Science+Business Media New York 2014

\begin{abstract}
For a positive integer $k$ we say that an association scheme with more than one point is $k$-equivalenced if each non-diagonal relation has valency $k$. In this paper we prove that every 3-equivalenced association scheme is Frobenius, that is, the set of relations coincides with the set of orbitals of a Frobenius group.
\end{abstract}

Keywords Association schemes $\cdot 3$-Equivalenced $\cdot$ Frobenius

\section{Introduction}

Let $G$ be a group acting transitively on a finite set $\Omega$. Then $G$ acts on $\Omega \times \Omega$ by

$$
(\alpha, \beta)^{g}=\left(\alpha^{g}, \beta^{g}\right),
$$

where $\alpha, \beta \in \Omega$ and $g \in G$. An orbit of the action of $G$ on $\Omega \times \Omega$ is called an orbital of $G$, and we denote the set of orbitals of $G$ by $S_{G}$. Then, each orbital $s \in S_{G}$ defines a digraph $(\Omega, s)$ whose out-degree is constant. We shall write the constant out-degree of $(\Omega, s)$ as $n_{s}$. Then, we have the following characterization:

M. Hirasaka $(\varangle) \cdot$ K.-T. Kim · J. R. Park

Department of Mathematics, College of Sciences, Pusan National University, 63 Beon-gil 2, Busandaehag-ro, Geumjung-gu, Busan 609-735, Korea e-mail: hirasaka@pusan.ac.kr

K.-T. Kim

e-mail: poisonn00@hanmail.net

J. R. Park

e-mail: parkjr@pusan.ac.kr 
(i) $G$ is 2-transitive on $\Omega$ if and only if $\left|S_{G}\right|=2$;

(ii) $G$ is $3 / 2$-transitive on $\Omega$ if and only if $n_{s}$ is constant on $s \in S_{G} \backslash\left\{1_{\Omega}\right\}$, where $1_{\Omega}=\{(\alpha, \alpha) \mid \alpha \in \Omega\}$

(iii) $G$ is regular on $\Omega$ if and only if $n_{s}=1$ for each $s \in S_{G}$.

An association scheme is a configuration consisting of a point set and a set of binary relations on the points with certain properties (see Sect. 2 for the details). For short we call it just a scheme for the remainder of this section. It is known (see [1, Ex. 2.1]) that $\left(\Omega, S_{G}\right)$ is a scheme. We say that a scheme $(\Omega, S)$ is schurian if $S=S_{G}$ for some permutation group $G$ on $\Omega$. In [1] we can see that schurian schemes play important roles in the theory of schemes or distance-regular graphs. But, a scheme is a purely combinatorial object just generalizing the orbitals of transitive permutation groups, and quite many non-schurian schemes are listed in [4]. For a positive integer $k$ we say that a scheme $(\Omega, S)$ with more than one point is $k$-equivalenced if $n_{s}=k$ for each $s \in S \backslash\left\{1_{\Omega}\right\}$, where $n_{s}$ is the valency of $s$, namely, the constant out-degree of $(\Omega, s)$. This is a combinatorial property generalizing $3 / 2$-transitivity. We say that $(\Omega, S)$ is regular (resp. Frobenius) if $S=S_{G}$ for some regular (resp. Frobenius) permutation group on $\Omega$.

It is an easy observation that the adjacency matrices of a 1-equivalenced scheme form a group. This implies that every 1-equivalenced scheme is regular (see [7, Theorem A]). However, the situation for $k$-equivalenced schemes with $k>1$ is completely different. In order to find non-trivial automorphisms from the property being $k$-equivalenced, we need to know some intersection numbers $\left\{c_{s s^{*}}^{t} \mid s, t \in S\right\}$ to determine the local structure of a non-diagonal relation (see Sect. 2 for the details). One may notice that the difficulty in determining the local structures from $\left\{c_{s s^{*}}^{t} \mid s, t \in S\right\}$ satisfying Lemma 2.1 (iv) with $u=s$ and $v=s^{*}$ is getting higher as $k$ increases. In the case of $k=2$, however, it works well and it is proved that every 2-equivalenced scheme is Frobenius (see [6]). In order to avoid repeating similar statements, we define $\mathcal{F}$ to be the set of positive integers $k$ such that every $k$-equivalenced scheme is Frobenius. According to the classification of schemes of small orders (see [4]), we find non-schurian $k$-equivalenced schemes with exactly three relations for $k=7,9,11,12,13,14,15$. Therefore,

\section{$7,9,11,12,13,14,15 \notin \mathcal{F}$.}

On the other hand, it is still open whether 4, 5, 6, 8, $10 \in \mathcal{F}$. However, in [5, Theorem 6.2], Muzychuk and Ponomarenko generalized the concept of pseudocyclic schemes for non-commutative ones and showed the following:

(i) A scheme $(\Omega, S)$ is pseudocyclic of valency $k$ if and only if $(\Omega, S)$ is $k$ equivalenced and, for each $t \in S \backslash\left\{1_{\Omega}\right\}$,

$$
\sum_{s \in S} c_{s s^{*}}^{t}=k-1
$$

(ii) Every Frobenius scheme is pseudocyclic;

(iii) Every pseudocyclic scheme $(\Omega, S)$ of valency $k$ is Frobenius if $|S| \geq 4(k-1) k^{3}$. 
The last one would be an immortal result in the theory of schemes to give a criterion for pseudocyclic schemes to be Frobenius though $|S| \geq 4(k-1) k^{3}$ is a rough bound, the first one shows that being $k$-equivalenced is quite weaker than pseudocyclicity, and the results suggest indirectly the following questions:

(i) Whether the bound $|S| \geq 4(k-1) k^{3}$ can be improved for small $k$;

(ii) Whether every $k$-equivalenced scheme $(\Omega, S)$ is pseudocyclic for small $k$;

(iii) Whether $k \in \mathcal{F}$ for small $k$.

The following gives the answer for the questions when $k=3$ :

Theorem 1.1 Every 3-equivalenced scheme is Frobenius.

In the case of $k=3$ we have two kinds of the local structure of a digraph $(\Omega, s)$ (see Lemma 3.2). It means that we need a slightly complicated argument in order to prove $3 \in \mathcal{F}$ in comparison with the case of $k=2$.

Obviously, if a $k$-equivalenced scheme of order $m$ exists, then $m \equiv 1(\bmod k)$, and the converse is true if $k=1$ (resp. 2), since a cyclic group of order $m$ (resp. a dihedral group of degree $m$ ) exists for each positive integer $m$. On the other hand, it is known (see [4]) that there is no 3-equivalenced scheme of order 10 or 22. Thus, it is natural to ask about the size $|\Omega|$ such that a 3-equivalenced scheme $(\Omega, S)$ exists, or equivalently, the degree $m$ such that a Frobenius group of order $3 m$ exists. This question can be solved by the following statement given in [2, Theorem 1]: a Frobenius group of degree $m$ and order $m k$ exists if and only if each maximal prime power which divides $m$ is congruent to one modulo $k$.

In Sect. 2 we prepare basic notation to make this paper as self-contained as possible. In Sect. 3 we give a proof of Theorem 1.1.

\section{Preliminaries}

We use the same notation on association schemes as in [5]. Let $\Omega$ be a finite set and $S$ a partition of $\Omega \times \Omega$. For $s \in S$ we denote $\{(\beta, \alpha) \mid(\alpha, \beta) \in s\}$ by $s^{*}$. We denote the set of $(\alpha, \alpha)$ with $\alpha \in \Omega$ by $1_{\Omega}$. For $\alpha \in \Omega$ and $s \in S$ we set

$$
\alpha s=\{\beta \in \Omega \mid(\alpha, \beta) \in s\} .
$$

We say that the pair $(\Omega, S)$ is an association scheme if it satisfies the following conditions (see [1], [7], and [8] for a background of the theory of association schemes):

(i) $1_{\Omega} \in S$;

(ii) For each $s \in S$ we have $s^{*} \in S$;

(iii) For all $u, v, w \in S$ the number $\left|\alpha u \cap \beta v^{*}\right|$ is constant whenever $(\alpha, \beta) \in w$. We denote the constant by $c_{u v}^{w}$.

For the remainder of this paper we assume that $(\Omega, S)$ is an association scheme, and we denote $S \backslash\left\{1_{\Omega}\right\}$ by $S^{\#}$.

For $\alpha, \beta \in \Omega$ we denote the unique element of $S$ which contains $(\alpha, \beta)$ by $r(\alpha, \beta)$. For $s \in S$ we denote $c_{s s^{*}}^{1}$ by $n_{s}$, and we call it the valency of $s$. 
Lemma 2.1 ([7]) For all $u, v, w \in S$ we have the following:

(i) $c_{u^{*} v}^{1_{\Omega}}=\delta_{u, v} n_{u}$, where $\delta$ is Kronecker's delta;

(ii) $c_{u v}^{w}=c_{v^{*} u^{*}}^{w^{*}}$;

(iii) $c_{u v}^{w^{*}} n_{w}=c_{v w}^{u^{*}} n_{u}=c_{w u}^{v^{*}} n_{v}$;

(iv) $n_{u} n_{v}=\sum_{s \in S} c_{u v}^{s} n_{s}$.

Applying Lemma 2.1 for a $k$-equivalenced association scheme $(\Omega, S)$ we obtain the following:

$$
k=\delta_{v, u^{*}}+\sum_{w \in S^{\#}} c_{u v}^{w} \text { for all } u, v \in S^{\#} .
$$

In [7] the complex product $U V$ of two subsets $U, V \subseteq S$ is defined to be

$$
U V=\left\{w \in S \mid \sum_{u \in U, v \in V} c_{u v}^{w}>0\right\} .
$$

We shall write $u V, U v$ instead of $U V$ if $U, V$ are singletons $\{u\},\{v\}$, respectively.

Lemma 2.2 For $u, v, w \in S$ with $w \in u v^{*}$ and $\alpha, \beta \in \Omega$ with $\beta \in \alpha u$ we have the following:

(i) $u^{*} v=\{r(\beta, \delta) \mid \delta \in \alpha v\}$;

(ii) $\beta$ has exactly $c_{w v^{*}}^{u^{*}}$ out-neighbors in $\alpha v$ with respect to $w$;

(iii) If $u=v$ and $w$ is symmetric but not diagonal, then $n_{u} c_{w u^{*}}^{u}$ is even.

Proof (i) Let $s \in u^{*} v$. Then $c_{u^{*} v}^{s}>0$. By Lemma 2.1 (iii), $c_{v s^{*}}^{u}=c_{u^{*} v}^{s} n_{s} / n_{u}>0$. Thus, there exists $\delta \in \alpha v \cap \beta s$, implying $s=r(\beta, \delta)$ with $\delta \in \alpha v$. Conversely, for $\delta \in \alpha v, r(\beta, \delta) \in r(\beta, \alpha) r(\alpha, \delta)=u^{*} v$.

(ii) follows from the definition of the constants $c_{u v}^{w}$.

(iii) follows since $\left|w \cap\left(\alpha u^{*} \times \alpha u^{*}\right)\right|=n_{u^{*}} c_{w u}^{u}=n_{u} c_{w u}^{u}$ by Lemma 2.1 (iii), and it is twice of the number of edges in the graph $(\alpha u,(\alpha u \times \alpha u) \cap w)$.

For $s \in S$ we define a matrix $A_{s}$ called the adjacency matrix of $s$ as follows:

$$
\left(A_{s}\right)_{\alpha \beta}:= \begin{cases}1 & \text { if } \quad(\alpha, \beta) \in s \\ 0 & \text { if } \quad(\alpha, \beta) \notin s\end{cases}
$$

where the rows and columns of $A_{s}$ are indexed by the elements of $\Omega$. It follows from the definition of association schemes that

$$
A_{u} A_{v}=\sum_{w \in S} c_{u v}^{w} A_{w}
$$

Recall that the Frobenius inner product on $\operatorname{Mat}_{\Omega}(\mathbb{C})$ is defined such that, for all $A, B \in \operatorname{Mat}_{\Omega}(\mathbb{C})$,

$$
\langle A, B\rangle=\frac{1}{|\Omega|} \operatorname{Tr}\left(A B^{*}\right),
$$


where $B^{*}$ is the Hermitian conjugate of $B$. As far as there is no confusion in the context we shall write just $u$ instead of $A_{u}$, and $u \cdot v$ instead of $A_{u} A_{v}$ in order to distinguish it from the complex product $u v$ for all $u, v \in S$. For example, we shall write (2) as

$$
u \cdot v=\sum_{w \in S} c_{u v}^{w} w
$$

The following lemma is frequently used in this paper. We shall use it with no additional mention.

Lemma 2.3 For all $u, v, w \in S$ we have the following:

(i) $\langle u, v\rangle=n_{u} \delta_{u, v}$;

(ii) $\langle u \cdot v, w\rangle=\left\langle u, w \cdot v^{*}\right\rangle=\left\langle v, u^{*} \cdot w\right\rangle=c_{u v}^{w} n_{w}$;

(iii) $\langle u \cdot v, u \cdot v\rangle=\left\langle u^{*} \cdot u, v \cdot v^{*}\right\rangle$.

Proof (i) follows from Lemma 2.1 (i), while (ii) and (iii) follow from the definition of $\langle *, *\rangle$ and (i).

Applying Lemma 2.3 for a $k$-equivalenced association scheme $(\Omega, S)$ we obtain from (1) that, for all $u, v \in S^{\#}$ with $v \neq u^{*}$,

$$
\begin{aligned}
\sum_{w \in S^{\#}}\left(c_{u v}^{w}\right)^{2} k & =\langle u \cdot v, u \cdot v\rangle=\left\langle u^{*} \cdot u, v \cdot v^{*}\right\rangle \\
& =k^{2}+\sum_{w \in S^{\#}} c_{u^{*} u}^{w} c_{v v^{*}}^{w} k \\
& \leq k^{2}+k \sum_{w \in S^{\#}} c_{u^{*} u}^{w} \sum_{w \in S^{\#}} c_{v v^{*}}^{w}=k^{2}+k(k-1)^{2}
\end{aligned}
$$

Since $c_{u u^{*}}^{w}<k$ for every $w \in S^{\#}$ by (1), this implies that, for every $k$-equivalenced association scheme $(\Omega, S)$ with $k>1$ and all $u, v, w \in S$,

$$
c_{u v}^{w}=k \Leftrightarrow v=u^{*} \neq 1_{\Omega} \text { and } w=1_{\Omega} .
$$

We use the same notation on group actions as in [3]. We denote the symmetric (resp. alternating) group on $\Omega$ by $\operatorname{Sym}(\Omega)(\operatorname{resp} . \operatorname{Alt}(\Omega))$. We define the automorphism group of $(\Omega, S)$ to be the set of $\sigma \in \operatorname{Sym}(\Omega)$ such that, for all $\alpha, \beta \in \Omega$,

$$
r(\alpha, \beta)=r\left(\alpha^{\sigma}, \beta^{\sigma}\right) .
$$

We denote it by $\operatorname{Aut}(\Omega, S)$. Suppose that $G$ is a transitive permutation group on $\Omega$. Then $G$ is contained in $\operatorname{Aut}\left(\Omega, S_{G}\right)$. Thus, in order to prove Theorem 1.1, it suffices to find a Frobenius group $G$ in the automorphism group of a 3-equivalenced association scheme $(\Omega, S)$ such that $S=S_{G}$. 


\section{Proof of the main result}

In this section we assume that $(\Omega, S)$ is a 3-equivalenced association scheme.

Lemma 3.1 For all $u, v \in S^{\#}$ with $u \neq v^{*}$ one of the following holds:

(i) $u \cdot v=w_{1}+w_{2}+w_{3}$ for some distinct $w_{1}, w_{2}, w_{3} \in S^{\#}$;

(ii) $u \cdot v=w_{1}+2 w_{2}$ for some distinct $w_{1}, w_{2} \in S^{\#}$.

Proof Applying (1) with $k=3$ we have

$$
\sum_{w \in S^{\#}} c_{u v}^{w}=3 .
$$

Since $c_{u v}^{w} \leq n_{w}=3$, it follows from (3) that $c_{u v}^{w} \leq 2$. This completes the proof.

Lemma 3.2 For every $u \in S^{\#}$ one of the following holds:

(i) $u \cdot u^{*}=3 \cdot 1_{\Omega}+w+w^{*}$ for some $w \in S$ with $w \neq w^{*}$;

(ii) $u \cdot u^{*}=3 \cdot 1_{\Omega}+2 u$ and $u=u^{*}$.

Proof Applying (1) with $k=3$ we have

$$
\sum_{w \in S^{\#}} c_{u u^{*}}^{w}=2
$$

Thus, we have either $c_{u u^{*}}^{w}=1$ or $c_{u u^{*}}^{w}=2$ for some $w \in S$. In the first case we have $w \neq w^{*}$ by Lemma 2.2 (iii), and $c_{u u^{*}}^{w}=c_{u u^{*}}^{w^{*}}$ by Lemma 2.1 (ii). Thus, (i) holds.

In the second case,

$$
u \cdot u^{*}=3 \cdot 1_{\Omega}+2 w .
$$

Since $c_{w u}^{u}=c_{u u^{*}}^{w}=2$ by Lemma 2.1 (iii), it follows from Lemma 2.2 (ii) with $k=3$ that the digraph $\left(\alpha u^{*}, w \cap\left(\alpha u^{*} \times \alpha u^{*}\right)\right)$ forms a triangle, especially, $w$ is symmetric. Thus, $w \in w w$ and $w=w^{*}$. It follows from Lemma 2.2 (iii) that $c_{w w}^{w}$ is even. Therefore,

$$
w \cdot w=3 \cdot 1_{\Omega}+2 w .
$$

Note that

$$
\langle w \cdot u, w \cdot u\rangle=\left\langle u \cdot u^{*}, w^{*} \cdot w\right\rangle=21 .
$$

If $u \neq w$, then the left-hand side is equal to 9 or 15 by Lemma 3.1, a contradiction. Therefore, (ii) holds.

Lemma 3.3 For all $u, v \in S^{\#}$, if $u=u^{*}$ and $u \in v v^{*}$, then $u=v$ and $1_{\Omega} \cup u$ is an equivalence relation on $\Omega$. 
Proof The assertion $u=v$ follows from Lemma 3.2. It is obvious that $1_{\Omega} \cup u$ is reflexive and symmetric. The equation as in Lemma 3.2 (ii) implies that $1_{\Omega} \cup u$ is transitive.

Lemma 3.4 For all $u, v, w \in S^{\#}$ with $v \neq w$ we have $\langle u \cdot v, u \cdot w\rangle \leq 9$, and equality holds if and only if $v \cdot w^{*}=2 s+s^{*}$, where $u^{*} \cdot u=3 \cdot 1_{\Omega}+s+s^{*}$ and $s \neq s^{*}$.

Proof Applying Lemma 3.1 and Lemma 3.2 to $v \cdot w^{*}$ and $u^{*} \cdot u$, respectively, we obtain from $v w^{*} \subseteq S^{\#}$ that

$$
\langle u \cdot v, u \cdot w\rangle=\left\langle v \cdot w^{*}, u^{*} \cdot u\right\rangle \in\{0,3,6,9,12\} .
$$

If $\langle u \cdot v, u \cdot w\rangle=12$, then $c_{u^{*} u}^{u}=c_{v w^{*}}^{u}=2$, implying that $u=u^{*}, u \cdot v=v+2 w$ and $u \cdot w=2 v+w$. Thus, $u^{*}=u \in v v^{*} \cap w w^{*}$. By Lemma 3.2, $u=v=w$, a contradiction. Therefore, $\langle u \cdot v, u \cdot w\rangle \leq 9$, and equality holds if and only if $u^{*} \cdot u=$ $3 \cdot 1_{\Omega}+s+s^{*}$ and $v \cdot w^{*}=2 s+s^{*}$, where $u^{*} \cdot u=3 \cdot 1_{\Omega}+s+s^{*}$ and $s \neq s^{*}$.

Lemma 3.5 Forall $u, v_{1}, v_{2}, v_{3} \in S^{\#}$, if $u \cdot v_{1}=u \cdot v_{2}=u \cdot v_{3}$, then $\left|\left\{v_{1}, v_{2}, v_{3}\right\}\right|<3$.

Proof Without loss of generality we may assume that $v_{1} \neq v_{2}$ and $v_{1} \neq v_{3}$. Since $u \cdot v_{1}=u \cdot v_{2}$, it follows from Lemma 3.4 that $v_{1} \cdot v_{2}^{*}=2 s+s^{*}$, where $u^{*} \cdot u=$ $3 \cdot 1_{\Omega}+s+s^{*}$ and $s \neq s^{*}$. By the same argument, we have $v_{1} \cdot v_{3}^{*} \in\left\{2 s+s^{*}, s+2 s^{*}\right\}$. Thus, $\left\langle v_{1} \cdot v_{2}^{*}, v_{1} \cdot v_{3}^{*}\right\rangle=15$ or 12 . By Lemma 3.4 , this forces $v_{2}=v_{3}$.

Fix $\alpha \in \Omega$. Then $\Omega$ is partitioned into $\{\alpha u \mid u \in S\}$. We define $\Gamma_{\alpha}$ to be the set of $\sigma \in \operatorname{Sym}(\Omega)$ such that $\sigma$ fixes only $\alpha$ and $(\alpha u)^{\sigma}=\alpha u$ for each $u \in S$. Remark that $\left|\Gamma_{\alpha}\right|=2^{|S|-1}$ since there are exactly two fixed-point-free permutations on each $\alpha u$ with $u \in S^{\#}$. For $\sigma \in \Gamma_{\alpha}$ and $u, v \in S^{\#}$ we shall write $u \sim_{\sigma} v$ if $r(\beta, \gamma)=r\left(\beta^{\sigma}, \gamma^{\sigma}\right)$ for each $(\beta, \gamma) \in \alpha u \times \alpha v$. It is clear that $\sigma$ is an automorphism of $(\Omega, S)$ if and only if $\sim_{\sigma}$ is the trivial relation on $S^{\#}$, that is, $u \sim_{\sigma} v$ for all $u, v \in S^{\#}$.

Lemma 3.6 For all $u, v \in S^{\#}$ and $\sigma \in \Gamma_{\alpha},\left\{(\alpha u \times \alpha v) \cap w \mid w \in u^{*} v\right\}$ is a partition of $\alpha u \times \alpha v$ which is invariant under exactly one of the following actions of $\sigma$ on $\alpha u \times \alpha v$ :

(i) $(\beta, \gamma)^{\sigma}=\left(\beta^{\sigma}, \gamma^{\sigma}\right)$ for each $(\beta, \gamma) \in \alpha u \times \alpha v$;

(ii) $(\beta, \gamma)^{\sigma}=\left(\beta^{\sigma}, \gamma^{\sigma^{-1}}\right)$ for each $(\beta, \gamma) \in \alpha u \times \alpha v$.

Moreover, $u \chi_{\sigma} v$ if and only if $r(\beta, \gamma)=r\left(\beta^{\sigma}, \gamma^{\sigma^{-1}}\right)$ for each $(\beta, \gamma) \in \alpha u \times \alpha v$.

Proof By Lemma 2.2 (i), $\left\{(\alpha u \times \alpha v) \cap w \mid w \in u^{*} v\right\}$ is a partition of $\alpha u \times \alpha v$. By Lemma 2.2 (i), (ii), $\sum_{w \in u^{*} v} c_{w v^{*}}^{u^{*}}=3$. Since $\left|u^{*} v\right| \in\{2,3\}$ by Lemma 3.1 and Lemma 3.2, there exists $w \in u^{*} v$ such that $c_{w v^{*}}^{u^{*}}=1$. Let $(\beta, \gamma) \in w$. Then $\beta^{\sigma} w \cap$ $\alpha v=\left\{\gamma^{\sigma^{i}}\right\}$ for a unique $i=1,2$ since $c_{w^{*} u^{*}}^{v^{*}}=c_{w v^{*}}^{u^{*}}=1$ by Lemma 2.1 (ii), (iii). Thus, we have

$$
(\alpha u \times \alpha v) \cap w=\left\{(\beta, \gamma),\left(\beta^{\sigma}, \gamma^{\sigma^{i}}\right),\left(\beta^{\sigma^{2}}, \gamma^{\sigma^{2 i}}\right)\right\}
$$


If $\left|u^{*} v\right|=2$, then we are done. If $\left|u^{*} v\right|=3$, then $c_{z v^{*}}^{u^{*}}=1$ for each $z \in u^{*} v$, and hence, by Lemma 2.2 (ii),

$$
\begin{aligned}
(\alpha u \times \alpha v) \cap r\left(\beta, \gamma^{\sigma}\right) & =\left\{\left(\beta, \gamma^{\sigma}\right),\left(\beta^{\sigma}, \gamma^{\sigma^{i+1}}\right),\left(\beta^{\sigma^{2}}, \gamma^{\sigma^{2 i+1}}\right)\right\}, \text { and } \\
(\alpha u \times \alpha v) \cap r\left(\beta, \gamma^{\sigma^{2}}\right) & =\left\{\left(\beta, \gamma^{\sigma^{2}}\right),\left(\beta^{\sigma}, \gamma^{\sigma^{i+2}}\right),\left(\beta^{\sigma^{2}}, \gamma^{\sigma^{2 i+2}}\right)\right\}
\end{aligned}
$$

This completes the proof of the first assertion since $\sigma^{2}=\sigma^{-1}$.

If $u \sim_{\sigma} v$, then, by the definition of $\sim_{\sigma},\left\{(\alpha u \times \alpha v) \cap w \mid w \in u^{*} v\right\}$ is invariant under the first action. By the first assertion, the contrapositive of "if" part of the second assertion holds. If $u \chi_{\sigma} v$, then $r\left(\beta_{0}, \gamma_{0}\right) \neq r\left(\beta_{0}^{\sigma}, \gamma_{0}^{\sigma}\right)$ for some $\left(\beta_{0}, \gamma_{0}\right) \in \alpha u \times \alpha v$. By the first assertion, $\left\{(\alpha u \times \alpha v) \cap w \mid w \in u^{*} v\right\}$ is invariant under the second action. This completes the proof of the "only if" part of the second assertion.

It is obvious that $\sim_{\sigma}$ is symmetric on $S^{\#}$, and $\sim_{\sigma}$ is reflexive by the following:

Lemma 3.7 For all $u \in S^{\#}$ and $\sigma \in \Gamma_{\alpha}$ we have $u \sim_{\sigma} u$.

Proof Since $1_{\Omega} \in u^{*} u$ by Lemma 2.1 (i),

$$
1_{\Omega}=r(\beta, \beta) \neq r\left(\beta^{\sigma}, \beta^{\sigma^{-1}}\right) \text { for } \beta \in \alpha u
$$

By the contrapositive of the third statement of Lemma 3.6, we have $u \sim_{\sigma} u$.

Lemma 3.8 Let $\sigma \in \Gamma_{\alpha}, u \in S^{\#}$ and $\beta \in \alpha u$. We shall denote the permutation $\left(\beta, \beta^{\sigma}\right) \sigma\left(\beta, \beta^{\sigma}\right)$ of $\Omega$ by $\sigma^{u}$. Then we have the following:

(i) $\sigma^{u}$ does not depend on the choice of $\beta \in \alpha u$;

(ii) $\sigma^{u} \in \Gamma_{\alpha}$;

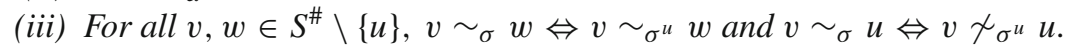

Proof It is a routine work to check that the action of $\sigma^{u}$ on $\Omega \backslash \alpha u$ coincides with that of $\sigma$, and the action of $\sigma^{u}$ on $\alpha u$ coincides with that of $\sigma^{2}$. This completes the proof of (i), (ii), and the former part of (iii).

Let $v \in S^{\#} \backslash\{u\}$. By Lemma 3.6, $v \sim_{\sigma} u$ if and only if $v \chi_{\sigma^{u}} u$. This completes the proof.

Lemma 3.9 Let $u \in S^{\#}$. Then there exists $\sigma \in \Gamma_{\alpha}$ such that $u \sim_{\sigma} v$ for all $v \in S^{\#}$.

Proof Choose $\sigma \in \Gamma_{\alpha}$ in such a way that $\left\{w \in S^{\#} \mid u \sim_{\sigma} w\right\}$ is maximal. Suppose that $\left\{w \in S^{\#} \mid u \sim_{\sigma} w\right\} \neq S^{\#}$. Then $u \chi_{\sigma} v$ for some $v \in S^{\#}$. By Lemma 3.7, $u \neq v$. Thus, by Lemma 3.8 (iii),

$$
\left\{w \in S^{\#} \mid u \sim_{\sigma^{u}} w\right\}=\left\{w \in S^{\#} \mid u \sim_{\sigma} w\right\} \cup\{v\}
$$

which contradicts the choice of $\sigma$.

Lemma 3.10 For each $\sigma \in \Gamma_{\alpha}$, the graph $\left(S^{\#}, \chi_{\sigma}\right)$ has no triangle. 
Proof Suppose to the contrary that $u \chi_{\sigma} v, v \chi_{\sigma} w$ and $w \chi_{\sigma} u$ for some distinct $u, v, w \in S^{\#}$ and $\sigma \in \Gamma_{\alpha}$. Since $\left|s t^{*}\right|=\left|t s^{*}\right| \in\{2,3\}$ for all distinct $s, t \in S^{\#}$ by Lemma 3.1, it suffices to show a contradiction for each of the following cases: (i) $\left|u^{*} v\right|=\left|v^{*} w\right|=2$; (ii) $\left|u^{*} v\right|=\left|v^{*} w\right|=3$.

(i) Suppose $\left|u^{*} v\right|=\left|v^{*} w\right|=2$. For $i=1,2$ there exists a unique $b_{i} \in u^{*} v$ $\left(c_{i} \in v^{*} w\right)$ such that $c_{u^{*} v}^{b_{i}}=i$ (resp. $c_{v^{*} w}^{c_{i}}=i$ ) by Lemma 3.1. Thus, $u^{*} v=\left\{b_{1}, b_{2}\right\}$ and $v^{*} w=\left\{c_{1}, c_{2}\right\}$. Let $(\beta, \gamma, \delta) \in \alpha u \times \alpha v \times \alpha w$ such that $(\beta, \gamma) \in b_{1}$ and $(\gamma, \delta) \in c_{1}$. By Lemma 2.1 (i), (ii), $c_{b_{2} v^{*}}^{u^{*}}=c_{u^{*} v}^{b_{2}}=2$ and $c_{c_{2}^{*} v^{*}}^{w^{*}}=c_{v^{*} w}^{c_{2}}=2$. Thus, by Lemma 2.2 (ii), $\beta$ has exactly two out-neighbors in $\alpha v$ with respect to $b_{2}$, and $\delta$ also has exactly two out-neighbors in $\alpha v$ with respect to $c_{2}^{*}$. Since

$$
\alpha v=\left\{\gamma, \gamma^{\sigma}, \gamma^{\sigma^{2}}\right\} \text { and } \gamma \in \beta b_{1} \cap \delta c_{1}^{*}
$$

it follows that

$$
\gamma^{\sigma}, \gamma^{\sigma^{2}} \in \beta b_{2} \cap \delta c_{2}^{*}
$$

Since $u \chi_{\sigma} v$ and $v \chi_{\sigma} w$, it follows from Lemma 3.6 that, for $i=0,1,2$,

$$
\left\{\gamma^{\sigma}, \gamma^{\sigma^{2}}\right\}^{\sigma^{-i}} \subseteq \beta^{\sigma^{i}} b_{2} \cap \delta^{\sigma^{i}} c_{2}
$$

By the uniqueness of $d$ with $c_{b_{2} c_{2}}^{d}=2, d=r\left(\beta^{\sigma^{i}}, \delta^{\sigma^{i}}\right)$ for $i=0,1,2$. By Lemma 3.6, $u \sim_{\sigma} w$, which contradicts $u \chi_{\sigma} w$.

(ii) Suppose $\left|u^{*} v\right|=\left|v^{*} w\right|=3$. By Lemma 3.1, we can take $d \in w^{*} u$ such that $c_{w^{*} u}^{d}=1$.

We claim that $d b=w^{*} v$ for each $b \in u^{*} v$. Let $(\beta, \gamma, \delta) \in \alpha u \times \alpha v \times \alpha w$ such that $(\delta, \beta) \in d$ and $(\beta, \gamma) \in b$. Applying Lemma 3.6 for $v \chi_{\sigma} w$ and $w \chi_{\sigma} u$ we obtain that, for $i=0,1,2$,

$$
d=r(\delta, \beta)=r\left(\delta^{\sigma^{i}}, \beta^{\sigma^{-i}}\right) \text { and } b=r(\beta, \gamma)=r\left(\beta^{\sigma^{-i}}, \gamma^{\sigma^{i}}\right) \text {. }
$$

Thus, $r\left(\delta^{\sigma^{i}}, \gamma^{\sigma^{i}}\right) \in r\left(\delta^{\sigma^{i}}, \beta^{\sigma^{-i}}\right) r\left(\beta^{\sigma^{-i}}, \gamma^{\sigma^{i}}\right)=d b$. Since $w \chi_{\sigma} v$, it follows from Lemma 3.6 with $\left|w^{*} v\right|=\left|v^{*} w\right|=3$ that $r\left(\delta^{\sigma^{i}}, \gamma^{\sigma^{i}}\right)$ with $\left.i=0,1,2\right\}$ are distinct. Since $\left\{r\left(\delta^{\sigma^{i}}, \gamma^{\sigma^{i}}\right) \mid i=0,1,2\right\} \subseteq w^{*} v$ by Lemma 2.2 (i), they are equal, and hence, $w^{*} v \subseteq d b$. Since $\left|w^{*} v\right|=3$ and $|d b| \leq 3$, the claim holds.

By the claim with $\left|w^{*} v\right|=3, d \cdot b=d \cdot b^{\prime}$ for all $b, b^{\prime} \in u^{*} v$. Since $\left|u^{*} v\right|=3$, it contradicts Lemma 3.5.

Proposition 3.11 For each $\sigma \in \Gamma_{\alpha}, \sim_{\sigma}$ is an equivalence relation on $S^{\#}$.

Proof From the definition of $\sim_{\sigma}$, it is clear that $\sim_{\sigma}$ is symmetric on $S^{\#}$. By Lemma 3.7, $\sim_{\sigma}$ is reflexive on $S^{\#}$. It remains to show that $\sim_{\sigma}$ is transitive on $S^{\#}$. Let $u, v, w \in S^{\#}$ such that $u \sim_{\sigma} v$ and $v \sim_{\sigma} w$. If $u \chi_{\sigma} w$, then $u, v$, and $w$ are distinct since $\sim_{\sigma}$ is reflexive. By Lemma 3.8 (iii), $u \chi_{\sigma^{v}} v, v \chi_{\sigma^{v}} w$, and $w \chi_{\sigma^{v}} u$, which contradicts Lemma 3.10. Therefore, $u \sim_{\sigma} w$. 
Lemma 3.12 If $\sigma \in \operatorname{Aut}(\Omega, S)$ fixes two distinct points in $\Omega$, then $|S|=2$ or $\sigma$ is the identity.

Proof Suppose $\alpha, \beta \in \Omega$ are distinct and fixed by $\sigma$. We set $u=r(\alpha, \beta)$ for short. Let $\alpha u=\left\{\beta_{0}, \beta_{1}, \beta_{2}\right\}$, where $\beta=\beta_{0}$. Suppose $|S|>2$. Then, there exists $v \in S^{\#}$ with $u \neq v$. By Lemma 3.1, there exists $w \in S^{\#}$ such that $c_{u^{*} v}^{w}=1$. Since $c_{v w^{*}}^{u}=$ $c_{u^{*} v}^{w}=1$ by Lemma 2.1 (iii), for $i=0,1,2$, there exists a unique $\gamma_{i} \in \alpha v \cap \beta_{i} w$. By $c_{u w}^{v}=c_{u^{*} v}^{w}=1, \gamma_{0}, \gamma_{1}$, and $\gamma_{2}$ are distinct. Since $\alpha$ and $\beta$ are fixed by $\sigma$, we have

$$
\left\{\gamma_{i}^{\sigma}\right\}=\alpha v \cap \beta_{i}^{\sigma} w \text { for } i=0,1,2 \text {, in particular, } \gamma_{0}^{\sigma}=\gamma_{0} \text {. }
$$

We claim that $\beta_{i}^{\sigma}=\beta_{i}$ for $i=1,2$. Suppose to the contrary that $\beta_{1}^{\sigma}=\beta_{2}$ and $\beta_{2}^{\sigma}=\beta_{1}$. Then

$$
r\left(\beta_{1}, \beta_{2}\right)=r\left(\beta_{1}^{\sigma}, \beta_{2}^{\sigma}\right)=r\left(\beta_{2}, \beta_{1}\right)=r\left(\beta_{1}, \beta_{2}\right)^{*} .
$$

By (4),

$$
r\left(\beta_{1}, \gamma_{0}\right)=r\left(\beta_{1}^{\sigma}, \gamma_{0}^{\sigma}\right)=r\left(\beta_{2}, \gamma_{0}\right)
$$

Thus,

$$
r\left(\beta_{1}, \beta_{2}\right) \in r\left(\beta_{1}, \gamma_{0}\right) r\left(\gamma_{0}, \beta_{2}\right)=r\left(\beta_{1}, \gamma_{0}\right) r\left(\beta_{1}, \gamma_{0}\right)^{*} .
$$

Applying Lemma 3.3 for $r\left(\beta_{1}, \beta_{2}\right) \in u^{*} u \cap r\left(\beta_{1}, \gamma_{0}\right) r\left(\beta_{1}, \gamma_{0}\right)^{*}$, we obtain that $r\left(\beta_{1}, \beta_{2}\right)=u=r\left(\beta_{1}, \gamma_{0}\right)$, and $u=r\left(\alpha, \gamma_{0}\right)$ since $1_{\Omega} \cup u$ is transitive, which contradicts $\gamma_{0} \in \alpha v$ since $u \neq v$.

By the above claim, $\sigma$ fixes each element of $\alpha u$. Moreover, by (4), $\sigma$ fixes each element of $\alpha v$. Since $v$ is arbitrarily taken from $S^{\#} \backslash\{u\}$, it follows that $\sigma$ fixes each element of $\Omega$.

Proof of Theorem 1.1 First, we claim that, for each $\alpha \in \Omega$,

$$
\Gamma_{\alpha} \cap \operatorname{Aut}(\Omega, S) \neq \emptyset .
$$

Applying Lemma 3.9 for an arbitrarily taken $u \in S^{\#}$ we obtain that there exists $\sigma \in \Gamma_{\alpha}$ such that $u \sim_{\sigma} v$ for all $v \in S^{\#}$. Applying Proposition 3.11 for $\sim_{\sigma}$ we obtain that $\sim_{\sigma}$ is the trivial relation on $S^{\#}$. This implies that $\sigma \in \operatorname{Aut}(\Omega, S)$.

We shall denote by $G$ the subgroup of $\operatorname{Sym}(\Omega)$ generated by

$$
\bigcup_{\alpha \in \Omega} \Gamma_{\alpha} \cap \operatorname{Aut}(\Omega, S) .
$$

By the first claim, $G_{\alpha} \cap \Gamma_{\alpha} \neq \emptyset$. This implies that $G_{\alpha}$ acts transitively on $\alpha s$ for each $s \in S$. 
We claim that $G$ is transitive on $\Omega$. Suppose $\alpha \in \Omega \backslash \beta^{G}$ for $\beta \in \Omega$. Then, for each $\gamma \in \beta^{G}, \alpha r(\alpha, \gamma)$ is contained in $\beta^{G}$ since it is an orbit of $G_{\alpha}$. Since $\left\{\alpha u \cap \beta^{G} \mid u \in S^{\#}, \alpha u \cap \beta^{G} \neq \emptyset\right\}$ is a partition of $\beta^{G}$, it follows that $\left|\beta^{G}\right| \equiv 0$ (mod 3). Therefore, we conclude that the size of each orbit of $G$ on $\Omega$ is divisible by 3 , which contradicts $|\Omega|=\sum_{s \in S} n_{s} \equiv 1(\bmod 3)$. Therefore, $G$ is transitive on $\Omega$.

By the second claim, $S$ is the set of orbitals of $G$ since $G_{\alpha}$ acts transitively on $\alpha s$ for each $s \in S$.

It remains to show that $G$ is Frobenius. If $|S|=2$, then $|\Omega|=4$ and $G=\operatorname{Alt}(\Omega)$, which is a Frobenius group. If $|S|>2$, then $G$ is Frobenius by Lemma 3.12.

Corollary 3.13 Let $(\Omega, S)$ be a 3-equivalenced scheme with $|S|>2$. Then $\operatorname{Aut}(\Omega, S)$ is a Frobenius group of order $3|\Omega|$.

Proof This is an immediate consequence of Theorem 1.1 and Lemma 3.12.

From now on we consider separability of 3-equivalenced schemes (see [5] for definitions of separability, coherent configurations, one-point fission $\left(\Omega, S_{\alpha}\right)$ of $(\Omega, S)$, fibers, semiregularity, and so on).

Lemma 3.14 Let $(\Omega, S)$ be a 3-equivalenced scheme with $|S|>2$ and $\alpha \in \Omega$. Then the coherent configuration $\left(\Omega, S_{\alpha}\right)$ is semiregular on $\Omega \backslash\{\alpha\}$.

Proof Let $S_{0}$ denote the set of orbitals of $\operatorname{Aut}(\Omega, S)_{\alpha}$. Since $\operatorname{Aut}(\Omega, S)_{\alpha}$ is semiregular on $\Omega \backslash\{\alpha\}$ by Corollary 3.13, the coherent configuration obtained from $\left(\Omega, S_{0}\right)$ by restricting to $\Omega \backslash\{\alpha\}$ is semiregular, i.e., $\left(\Omega, S_{0}\right)$ is semiregular on $\Omega \backslash\{\alpha\}$. Moreover, $S_{\alpha}$ is a fusion of $S_{0}$, i.e., each element of $S_{\alpha}$ is a union of elements of $S_{0}$. For $u, v \in S^{\#}$ we shall write the set of elements of $S_{\alpha}$ (resp. $S_{0}$ ) contained in $\alpha u \times \alpha v$ as $S_{\alpha}(u, v)$ (resp. $\left.S_{0}(u, v)\right)$. We define a binary relation $\sim$ on $S^{\#}$ such that $u \sim v$ if and only if $S_{0}(u, v)=S_{\alpha}(u, v)$.

First, we claim that $S_{\alpha}(u, v) \cap S_{0}(u, v) \neq \emptyset$ for all $u, v \in S^{\#}$ and $u \sim v$ whenever $\left|u^{*} v\right|=3$. Since $\left|u^{*} v\right| \in\{2,3\}$ by Lemma 3.1 and 3.2 and $S_{\alpha}$ is a fusion of $S_{0}$, it follows from Lemma 2.2 that $\alpha u \times \alpha v$ contains at least one element (resp. three elements) of $S_{\alpha} \cap S_{0}$ as a subset when $\left|u^{*} v\right|=2$ (resp. $\left|u^{*} v\right|=3$ ). Since $\left|S_{0}(u, v)\right|=3$, we have $S_{\alpha}(u, v)=S_{0}(u, v)$ whenever $\left|u^{*} v\right|=3$.

Second, we claim that, if $u \sim v$ for some $v \in S^{\#}$, then $u \sim w$ for all $w \in S^{\#}$. By the first claim, there exists $s \in S_{\alpha}(v, w) \cap S_{0}(v, w)$. Since $\left(\Omega, S_{0}\right)$ is semiregular on $\Omega \backslash\{\alpha\}$ and $S_{\alpha}(u, v)=S_{0}(u, v)$, the products $t s$ with $t \in S_{\alpha}(u, v)$ are distinct singletons in $S_{\alpha}(u, w) \cap S_{0}(u, w)$, where the product $t s$ on a coherent configuration is defined by the similar way to the complex product on a scheme (see the last sentence of [5, Sect. 2.1]). Therefore, $S_{\alpha}(u, w)=S_{0}(u, w)$.

Third, we claim that $\sim$ is not empty. Let $u, v \in S^{\#}$ with $u \neq v$. If $u \neq u^{*}$, then $\left|u^{*} u\right|=3$ by Lemma 3.2, which implies $u \sim u$ by the first claim. If $u=u^{*}$, then by Lemma 3.1,

$$
\langle u \cdot v, u \cdot v\rangle=\left\langle u^{*} \cdot u, v \cdot v^{*}\right\rangle=9+2 \cdot 3 \cdot c_{v v^{*}}^{u},
$$

which is equal to 9 since $c_{v v^{*}}^{u}=0$ by Lemma 3.1. By Lemma 3.2, $\left|u^{*} v\right|=|u v|=3$, which implies $u \sim v$ by the first claim. 
Since $\sim$ is symmetric by the definition of coherent configurations and $\sim$ is transitive by the second claim, it follows from the second and third claims that $\sim$ is the trivial relation. Therefore, $S_{0}=S_{\alpha}$.

Corollary 3.15 Every 3-equivalenced scheme is separable.

Proof The assertion is obvious if $|S|=2$. If $|S|>2$, Lemma 3.14 shows that the assumption of [5, Theorem 7.2] is satisfied. Therefore, the assertion follows from [5, Theorem 7.2].

Acknowledgments The first author would like to express his deepest gratitude to Professor Akihiro Munemasa for his careful reading and valuable comments in writing the paper. Also, the authors would like to thank anonymous referees for their suggestions to add the last two corollaries. This work was supported by a 2-Year Research Grant of Pusan National University, by the BK21 and by the NRF.

\section{References}

1. Bannai, E., Ito, T.: Algebraic Combinatorics. I. Association Schemes. The Benjamin/Cummings Publishing Co., Inc., Menlo Park (1984)

2. Boykett, T.: Construction of Ferrero pairs of all possible orders. SIAM J. Discret. Math. 14(3), 283-285 (2001)

3. Dixon, J.D., Mortimer, B.: Permutation Groups, Graduate Texts in Mathematics, vol. 163. Springer, New York (1996)

4. Hanaki, A., Miyamoto, I.: Classification of association schemes with small verticies. http://kissme. shinshu-u.ac.jp/as/ Accessed 2013

5. Muzychuk, M., Ponomarenko, I.: On pseudocyclic association schemes. Ars Math. Contemp. 5(1), 1-25 (2012)

6. Muzychuk, M., Zieschang, P.-H.: On association schemes all elements of which have valency 1 or 2. Discret. Math. 308(14), 3097-3103 (2008)

7. Zieschang, P.-H.: An Algebraic Approach to Association Schemes. Lecture Notes in Mathematics, vol. 1628. Springer, Berlin (1996)

8. Zieschang, P.-H.: Theory of Association Schemes. Springer Monograph in Mathematics. Springer, Berlin (2005) 